\title{
BMJ Open Models of care for patients with knee osteoarthritis in primary healthcare: a scoping review protocol
}

\author{
Daniela Costa (10 , 1,2,3 Eduardo B Cruz (D) , ${ }^{2,4}$ Ana M Rodrigues, ${ }^{2,3}$ \\ Daniela Gonçalves-Bradley, ${ }^{5}$ Luís A Gomes, ${ }^{2,3,4}$ Helena Donato (D) , ${ }^{6}$ Carla Nunes ${ }^{1,2}$
}

To cite: Costa D, Cruz EB, Rodrigues AM, et al. Models of care for patients with knee osteoarthritis in primary healthcare: a scoping review protocol. BMJ Open 2021;11:e045358. doi:10.1136/ bmjopen-2020-045358

- Prepublication history and additional online supplemental material for this paper are available online. To view these files, please visit the journal online. To view these files, please visit the journal online (http://dx.doi.org/10.1136/ bmjopen-2020-045358).

Received 28 September 2020 Accepted 25 May 2021

Check for updates

(c) Author(s) (or their employer(s)) 2021. Re-use permitted under CC BY-NC. No commercial re-use. See rights and permissions. Published by BMJ.

For numbered affiliations see end of article.

Correspondence to

Daniela Costa;

dcosta.ft@gmail.com

\section{ABSTRACT}

Introduction Knee osteoarthritis (OA) is a prevalent condition with associated high disability and healthcare costs. Evidence of major gaps in the implementation of evidence-based interventions in people with knee OA led several healthcare systems to implement models of care (MoCs) in order to improve knowledge translation and guaranty their economic sustainability. Nevertheless, there are few studies that analyse the existing body of evidence of MoCs for patients with knee $0 \mathrm{~A}$ in primary healthcare settings. Therefore, we aim to identify MoCs developed for patients with knee $0 \mathrm{~A}$ implemented in primary healthcare and, analyse their core components and outcomes.

This scoping review will create knowledge about the components and outcomes of these MoCs which, in the future, will facilitate their transferability to practice. Methods and analysis We will include studies that developed and implemented an MoC for people with knee $O A$ in primary healthcare. We will use the PCC mnemonic, being 'Population'-people with Knee OA, 'Concept' - the MoCs and 'Context' - the primary healthcare setting. We will conduct the search on PubMed, EMBASE, Cochrane Central Register of Controlled Trials, Scopus, Web of Science Core Collection, as well as grey literature databases and relevant institutions and organisations websites, for articles published after 2000. Two independent reviewers will screen titles and abstracts followed by a full-text review to assess papers regarding their eligibility. We will evaluate the methodological quality of the included studies with the Mixed Methods Appraisal tool and apply a data abstraction form to describe and interpret the evidence.

Ethics and dissemination As a secondary analysis, this scoping review does not require ethical approval. Findings will be published in peer-review journal, presented in scientific conferences and as a summary through primary healthcare units.

\section{INTRODUCTION}

Osteoarthritis (OA) affects approximately 250 millions of people worldwide, ${ }^{1}$ and is responsible for 9.6 million years lived with disability. ${ }^{2}$ Alongside the impairments in physical function, ${ }^{3}$ people with OA often experience chronic pain, depression and sleeping problems. ${ }^{4}$ Worldwide, healthcare costs related to OA represent approximately $0.9 \%$ of national

\section{Strengths and limitations of the study}

To our knowledge, this is the first scoping review that aims to map the literature about models of care developed and implemented for patients with knee osteoarthritis.

- We will undertake a comprehensive search strategy for published and unpublished studies in peer-reviewed journals databases, grey literature databases, handsearch in relevant journals, organisation and institution websites and conference proceedings, with the support of a research librarian.

- This scoping review is limited to articles written in English, Spanish and Portuguese, since 2000.

- The quality appraisal of the included studies, although not common on scoping reviews, will provide a better interpretation of the results and will identify the gaps in evidence in this topic.

- This scoping review will generate hypothesis for the development of new models of care and support future intervention research studies.

healthcare systems, ${ }^{5}$ representing 1\%-2.5\% of the Gross Domestic Product (GDP) of high-income countries. ${ }^{6}$

The knee is the most affected joint and it is responsible for approximately $85 \%$ of the burden of OA. ${ }^{2}$ The worldwide increase of obesity, alongside with the proportion of people with low levels of physical activity, are associated with the rising prevalence and incidence of knee OA. ${ }^{7}$

There is no known cure for OA. Currently, evidence-based approaches aim to improve joint function and patients' quality of life, relieve pain and modify risk factors for disease progression. Clinical practice guidelines recommend exercise, education, self-management and healthy weight maintenance as core interventions for knee OA, during all stages of this disease. Pharmacological management is recommended for symptom control, and total knee arthroplasty (TKA) is considered a latter option, when quality of life is significantly impaired, even 
with the implementation of conservative interventions. ${ }^{89}$ Although TKA is considered to be a cost-effective intervention, ${ }^{10}$ patient-reported outcomes highlight its shortcomings. Evidence reveals that $5 \%$ of patients report postoperative complications, ${ }^{11} 50 \%$ report adverse events in the first year after surgery, ${ }^{12} 20 \%-34 \%$ report dissatisfaction with surgery, ${ }^{13}$ and $3 \%-5 \%$ need surgery revision in less than 10 years. ${ }^{14}$ Furthermore, the mortality rate associated with TKA is approximately $0.2 \%-0.37 \% .^{15}$

Despite international recommendations, evidence across several countries report that conservative nonpharmacological interventions are offered to less than $50 \%$ of patients. ${ }^{16}{ }^{17}$ Moreover, TKA incidence rate has been rising exponentially in the Organisation for Economic Co-operation and Development (OECD) countries, for patients both above and below 65 years old. The reported annual growth rate of $5.5 \%$ is disproportional even when the epidemiological changes are taken into account. ${ }^{18}$ Furthermore, qualitative studies reveal that healthcare professionals see $\mathrm{OA}$ as a trivial condition with an unavoidable progression, and often believe that conservative treatments lack efficacy. ${ }^{19}$

Taking this data into account, there are major gaps in the translation of evidence to practice, and the care of patients with $\mathrm{OA}$ has been characterised by symptomdriven and segmented interventions ${ }^{20}$ centred on pharmacological, ${ }^{16}{ }^{17}$ surgical options. ${ }^{21}$ The complex and multidomain target for knee OA treatment can only be achieved by person-oriented intervention models that are multidisciplinary, if needed, and include a timely integration of evidence-based interventions. ${ }^{20}$ This knowledge has driven national health policies in several countries to create strategies to improve intervention outcomes, guarantee the sustainability of healthcare systems and the costeffectiveness of interventions of patients with $\mathrm{OA}^{22}$ These strategies encompass the implementation of models of care (MoCs) that are used as facilitators to bridge the gap between evidence and care delivery and practice, by describing not only the principles of care for a given condition (what to do), but also to guide how these principles can be implemented in a local setting (the 'how') ${ }^{23}$

MoCs for musculoskeletal diseases are showing promising results in overall patient satisfaction with care, ${ }^{24}$ improvement of patient-level outcomes and adherence to guidelines recommendations, ${ }^{25}$ and appropriate use of medication and reduction of absence from work. ${ }^{26}$

Several MoCs for OA have been implemented and published worldwide, ${ }^{24-27}$ mainly in primary healthcare settings. Usually, MoCs are complex interventions thus, its transferability into practice is challenging due to their context dependency. Moreover, in 2018 a group of researchers and clinicians established the 'Joint Effort Initiative', endorsed by Osteoarthritis Research Society International, which mission includes the investigation of the most effective OA Management Programmes, that are MoCs for OA, and the development of long-term strategies for effective implementation in different socioeconomic and cultural environments. The first action of this initiative was to identify and prioritise future work, with a survey with delegates with known interest on OA Management Programmes. The highest priority ranked action statement identified was 'to establish guidelines for the implementation of different OA Management Programmes to ensure consistency of delivery and adherence to international best practice MoC'. ${ }^{28}$ Therefore, creating knowledge of and clarifying which intervention components have been included in the MoCs, as well as exploring their outcomes is paramount and needed worldwide.

Few studies have synthesised evidence on MoCs for OA. The narrative review by Allen $e t a l^{2929}$ shares some preliminary evidence about the characteristics of selected MoCs designed for patients with OA. This is, to our knowledge, the only study that synthesised the evidence on this topic. This review identified several MoCs for prevention, management in the disease continuum and specific for advanced OA, namely directed for total joint replacement surgery. The authors concluded that there are emerging efforts in multiple countries to implement MoCs, mostly focused on non-pharmacological interventions. Additionally, it is concluded that there is a need to examine the impact of these MoCs, and to explore how this MoCs can be adapted and implemented in other contexts. ${ }^{29}$ Due to its inherent methodological limitations, such as the absence of a search strategy, methodological quality assessment of the included studies and a standardised method for data extraction, it is not possible to replicate this review nor to understand how studies were selected. Notwithstanding its importance, this study provides a biased depiction of the literature of MoCs developed for patients with OA.

With this scoping review, we aim to map the existing literature of MoCs developed for people with knee OA through a systematic methodology. Our primary objective is to identify MoCs developed for patients with knee $\mathrm{OA}$ in primary healthcare and describe their components. The secondary objectives are to describe the outcomes of MoCs and to identify specific aspects of the context reported related with the implementation of the MoC. These context-related aspects, which can influence the success and transferability of the MoCs, will be divided in three categories: external (e.g., supportive national/local policies, governmental financial incentives, dominant paradigms in society as evidence-based practice or patient-centred care, support of stakeholders), organisational (e.g., presence of a culture of innovation, leadership characteristics, organisational readiness, resources available and professional relationships among team and patients) and population-level (e.g., specific geographic areas with different access to health services, subpopulations with special sociodemographic and clinical characteristics). ${ }^{30}$ Although qualitative assessment is not usually performed on scoping reviews, ${ }^{3132}$ we will include it to describe the quality of research in this field. 


\section{METHODS AND ANALYSIS}

A scoping review is a valid, comprehensive and transparent method for mapping the body of evidence underpinning a research area, main sources and type of evidence available. Scoping reviews are mainly made for complex and heterogeneous research topics or for those which were not previously comprehensively reviewed. ${ }^{32}{ }^{33}$ Given the stated objectives of this review, the topic of interest is the identification, mapping, reporting and discussion of the characteristics of MoCs. Therefore, our purpose is better aligned with a scoping review, instead of systematic review, considering the criteria described by Munn $e t a l^{34}$

The methodology of this scoping review will be guided by established methodological frameworks, as outlined by Arksey and $\mathrm{O}^{\prime} \mathrm{Malley}^{33}$, and enhanced by Levac $e a^{31}$ and Joanna Briggs Institute (JBI) ${ }^{35}$ The Preferred Reporting Items for Systematic Reviews and Meta-Analysis extension (PRISMA) for Scoping Reviews will guide the development of the scoping review to ensure transparency of the results uptake. ${ }^{36}$ To our knowledge, and after a search in relevant databases, this is the first protocol for a scoping review that aim to identify the MoCs developed and implemented for people with knee OA.

\section{Research questions}

According to the objectives previously formulated, this review will attain to answer the following research questions

1. Which MoCs have been implemented for noninstitutionalised patients with knee OA attending primary healthcare units?

2. What are the core components included in the MoCs (these include, but are not limited to, interventions and their characteristics, healthcare professionals involved, programmes duration, funding)?

3. What are the MoCs outcomes and how have they been measured (patient-level outcomes, system-level outcomes and implementation outcomes)?

4. Which are the aspects of the included MoCs that are context-specific at external, organisational and population level (as described in the introduction section)?

\section{Identification of the relevant literature}

\section{Inclusion criteria}

To guide the identification and inclusion of the studies we will use the mnemonic 'PCC' referring to Population, Concept and Context, as proposed by JBI. ${ }^{35}$

\section{Population}

We will consider studies that included non-institutionalised adults (that live in the community, and are not residents/ inpatients in any institution, like hospitals, psychiatric centres, nursing homes, military institutions or prisons), diagnosed with knee OA who have not undergone, or scheduled, TKA. Studies that involve patients with other types of arthritis or patients with $\mathrm{OA}$ in multiple joints will be included if more than $50 \%$ of the patients have diagnosis of knee OA.

\section{Concept}

The concept of interest in this review is MoCs designed for patients with knee OA. In this study, we will use the definition by Briggs et al that defines an MoC as 'an evidence-informed policy or framework that outlines the optimal manner in which condition-specific care should be made available and delivered to consumers at a system level'. ${ }^{23}$ Therefore, 'a MoC aims to describe the principles of care for a given condition (the 'what') as well as guidance on how those principles could be implemented in a local setting (the 'how')'. A distinction should be made between MoCs, clinical guidelines and models of service delivery. MoC serve as guides that complement clinical practice guidelines, describing how best evidence can be implemented as a sector-wide model of service delivery by clinicians, consumers and health systems across the disease continuum, tailored to the specificities of local context. Thus, a model of service delivery converts the principles of an MoC into operational activity and operational recommendations, relevant to the local context, modes of service delivery and evaluation, considering resources, infrastructure and workforce capacity requirements. As mentioned, the concept of interest of this scoping review is $\mathrm{MoC}$, and we will only consider the model of service delivery of a specific MoC to describe its organisational components. ${ }^{23}$

To guide the inclusion of the studies in this review we develop operational a priori criteria to distinguish a model of care from other types of interventions based on The Framework to Evaluate Musculoskeletal MoC, Briggs et $a \hat{l}^{37}$ and Eyles et $a l^{28} .{ }^{23} 2837$ Only studies that address the implementation in a real-world setting of MoC's and that fulfil all the following criteria will be considered for inclusion:

- Defines the optimal care manner to deliver care for people with $\mathrm{OA}$, with an underlying evidenceinformed strategy, framework or pathway.

- Describes the operationalisation of the MoC, for example, who deliver care, when and where care is best delivered and how it is to be delivered.

- The MoC is tailored according to context.

- Care is coordinated, with longitudinal progression and reassessments.

- The implementation of the MoC has patient-level, organisational-level and/or implementation-level goals.

In this scoping review, we will consider the identified MoC as the unit of interest thus, several studies that report the outcomes of the same MoC will be considered.

According to the stated research questions as well as the recommendations for implementation of MoC's, ${ }^{37}$ we will a priori include studies that describe, but are not limited to: (1) Which MoC was implemented: identification of MoC and/or frameworks/theory used; (2) What and how care is provided, and by whom: underlying interventions at patient level, professionals training, services involved, organisation of care, among others; (3) How the MoC is assessed and what were its outcomes at patient's 
level (e.g., pain, function or quality of life, satisfaction, collected with self-reported questionnaires or interview questionnaires or performance measures), at system level: (e.g., rate of referral or prescription for exercise, rate of prescribed exams, healthcare costs, waiting times, quality indicators, perceptions/perspectives of stakeholders) and implementation outcomes (e.g., acceptability, adoption, feasibility, fidelity, penetration, sustainability) and (4) What are the aspects, if any, of the MoC that are contextspecific at external, organisational and population level, as described in introduction section. We will include MoCs that consider non-pharmacological interventions (for example, exercise, patient education, behavioural change programmes, self-efficacy and self-management programmes), combined or not with pharmacological interventions, integrated in an MoC. We will exclude MoCs that focus only on pharmacological interventions, or that includes surgery or complementary and alternative medicine interventions.

\section{Context}

This review will be focused on primary healthcare context. WHO defines primary healthcare as a 'wholeof-society approach to health'. ${ }^{38}$ According with guidelines recommendations, primary care is the most relevant setting worldwide for both research and management of patients with $\mathrm{OA}$ for prevention and across the disease continuum. $^{89}$

We will include studies that have implemented MoCs focused on primary care services, but we will consider also MoCs that include interventions at other levels of healthcare delivery as long as it includes primary care services in the patient's pathway. The inclusion of primary healthcare in the pathway may be referred as the first contact with the healthcare system, for direct provision of care, point of referral to other levels of care or services or continuity of care. Primary care services may cover all the full continuum of health promotion and disease prevention, treatment and rehabilitation, delivered at individual or community-oriented approach. Thus, MoCs that consider in the pathway services such as, but not limited to, community pharmacies, physiotherapy and nutrition outpatient services, physical activity community services as well as hospitals or other secondary care settings linked with primary care will be included. ${ }^{38}$

\section{Types of sources}

We will include quantitative studies with comparative (randomised controlled, cohort, quasi-experimental) and non-comparative methods (narrative, policy reports, audit) related with the implementation of MoCs for patients with knee OA, that report outcomes of the implementation of the MoC. Qualitative or mixed-methods study designs will also be considered only if they report outcomes of the implementation for example, qualitative evaluation studies.

We will exclude studies that aim to test clinical effectiveness or efficiency of specific clinical interventions that do not undertake implementation interventions. In order to distinguish typical clinical intervention trials and implementation intervention trials related to MoCs (e.g., pilot studies, cluster or pragmatic randomised controlled trials) during title and abstract screening, we will consider studies for full text screening when the experimental group is compared with usual care, at the same or at another healthcare unit, wait list or no treatment whenever the title and abstract suggests an underlying MoC. ${ }^{39}$ We will assume overinclusion of clinical intervention studies for full-text screening whenever doubts arise.

Literature published between 2000 and 2020 written in English, Portuguese or Spanish, in peer-reviewed and grey literature will be included in order to capture the most recent evidence on the implementation of MoC aligned with current paradigms of healthcare delivery. ${ }^{40}$

\section{Search strategy}

We will run the search query in PubMed, EMBASE and Cochrane Central Register of Controlled Trials (CENTRAL), Scopus (including conference proceedings), Web of Science Core Collection (including conference proceedings). Then, we will conduct a hand search in relevant peer-reviewed journals: Osteoarthritis and Cartilage, Best Practice and Research Clinical Rheumatology, BMC Musculoskeletal Disorders, Arthritis Care and Research, Implementation Science, Health Services and Delivery Research, JBI Evidence Synthesis and BMC Health Services Research.

We will perform the search in Open Grey, Grey Literature Report databases and MedNar search engine to identify grey literature records. We will also search websites of relevant institutions and organisations, such as WHO, Arthritis Australia, American College of Rheumatology, Osteoarthritis Research Society International, European League Against Rheumatism, Agency for Clinical Innovation Musculoskeletal Network, Arthritis Community Research \& Evaluation Unit, National Institute for Health Research, and Bone and Joint Initiative.

We will screen the reference lists of the selected articles for inclusion to identify additional potentially eligible primary studies. Additionally, we will check reference lists of previous published reviews. We will contact authors of the included studies to clarify any questions we might have about their published reports and to seek unpublished data related to the MoC. Different reports of the same MoC will be collated, as each MoC is the unit of interest. ${ }^{41}$

We undertook a preliminary search in PubMed in September of 2020, which was built according to the 'PCC' mnemonic. The search terms included 'osteoarthritis', 'models of care' and 'primary care' and their synonyms, in title or abstract. We used the text words of relevant articles on this topic and the index terms used to describe these articles to develop a full search strategy (online supplemental file). As MoC are often poorly defined in the literature, and used interchangeably, as 'service improvement frameworks', 'models of service 
delivery', 'clinical frameworks' or 'care pathways', we will use a broad search strategy to cover all relevant studies. We will adapt the search strategy to each of the included databases. This process will be held in collaboration with a research librarian. We will conduct the searches from May to June 2021.

\section{Study selection}

Two independent reviewers (DC and LAG) will screen abstracts and titles of the search records. We will adopt maximum sensitivity during title and abstract screening, and retrieve full texts for all records included by at least one reviewer. The review team will perform a pilot testing of source selectors with the selection of a sample of 25 random titles/abstracts. We will screen this sample using the defined eligibility criteria, discussing discrepancies and making any necessary modifications. Screening will start only when we achieve an agreement of $75 \%$ or more. ${ }^{41}$ For full-text screening we will note specific reasons for exclusion.

All identified articles will be collated and uploaded, duplicates will be removed, potentially relevant papers will be extracted in full and their citation details will be uploaded using EndNote X7.8 (Clarivate Analytics, USA). We will report a narrative description of the process of the search in the final scoping review accompanied by a PRISMA flow diagram. ${ }^{36}$

As previously stated, the unit of interest is the identified MoC, therefore we expect that, during the study selection, we will identify more than one study for the same MoC. Thus, all papers with a MoC that respects the inclusion criteria for population and context will be investigated for additional papers and then aggregated. In the end of full-text screening, we will collate information of different single studies related with the same MoC.

\section{Data charting}

Two reviewers (DC and LAG) will independently complete a standard data abstraction form to extract data from each study using a pilot-tested data abstraction form. Charting is a technique for organising and interpreting the data by screening, charting and sorting material according to the main issues and themes. The reviewers will apply the pilot-test data abstraction form in at least five articles to test and ensure consistency of the form in line with the purpose of the study, as presented in table 1. Changes will be made and discussed with the team prior to extracting data from the remaining articles. As an iteractive process, during the data extraction some items can be added according with studies found.$^{32}$ We will detail these modifications in full in the scoping review.

\section{Quality assessment}

Performing quality assessment is not common and is also a controversial issue in scoping reviews. The absence of quality appraisal is usually referred as a methodological limitation, ${ }^{42}$ and, at the same time, a necessary component. ${ }^{43}$ By qualitatively appraising the included studies,
Table 1 Data extraction according to scoping review research questions

\begin{tabular}{|c|c|}
\hline & Data to be extracted \\
\hline \multirow[t]{6}{*}{ Summary } & Authors \\
\hline & Title \\
\hline & Year of publication \\
\hline & Citation \\
\hline & $\begin{array}{l}\text { Source of Information (peer-review or } \\
\text { grey literature) }\end{array}$ \\
\hline & Study designs \\
\hline \multirow{3}{*}{$\begin{array}{l}\text { Research question } \\
1 \text { : identification of } \\
\text { the MoC }\end{array}$} & MoC Identification (name, if applicable) \\
\hline & Country \\
\hline & $\begin{array}{l}\text { Population addressed and sample size, } \\
\text { including proportion of participants } \\
\text { with Knee OA ( } \mathrm{n} / \% \text { of total) }\end{array}$ \\
\hline \multirow{3}{*}{$\begin{array}{l}\text { Research } \\
\text { question 2: core } \\
\text { components }\end{array}$} & Theory/framework \\
\hline & $\begin{array}{l}\text { Interventions components and their } \\
\text { characteristics }\end{array}$ \\
\hline & $\begin{array}{l}\text { Organisational components (eg, } \\
\text { healthcare professionals involved, } \\
\text { workforce capacity, programmes } \\
\text { duration, funding, care coordination) }\end{array}$ \\
\hline \multirow{3}{*}{$\begin{array}{l}\text { Research question } \\
3: \text { outcomes and } \\
\text { outcome measures } \\
\text { used }\end{array}$} & $\begin{array}{l}\text { Outcomes addressed and outcome } \\
\text { measures }\end{array}$ \\
\hline & $\begin{array}{l}\text { Main results of MoC (outcomes at } \\
\text { organisation and patient level }\end{array}$ \\
\hline & $\begin{array}{l}\text { Evaluation of implementation success } \\
\text { (if applicable) }\end{array}$ \\
\hline \multirow{3}{*}{$\begin{array}{l}\text { Research question } \\
4: \text { context-specific } \\
\text { components }\end{array}$} & Context-specific external factors \\
\hline & Context-specific organisational factors \\
\hline & Context-specific population factors \\
\hline
\end{tabular}

MoC, models of care; OA, osteoarthritis.

we will be able to map the quality of the literature in this field. Therefore, not only will we identify where research is lacking, but also the gaps in evidence base, identify the types of available evidence, and how research has been conducted in this area. ${ }^{34}$ Additionally, quality appraisal is of paramount importance for the interpretation and dissemination of the results of this scoping review, enhancing their usefulness to practice, policy-making and for future research. ${ }^{44}$

Two independent reviewers will analyse the methodological quality of the retrieved studies using the Mixed Methods Appraisal Tool, 2018 version, since we expect to find a wide range of study types. This is a validated tool to evaluate qualitative research, randomised controlled trials, non-randomised studies, quantitative description studies and mixed-methods studies. Any disagreements between reviewers will be solved by consensus or decision of a third reviewer. We will report results in a narrative form and in a table that will contain the accomplishment information of each item of the checklists, described as 
'yes', 'no' and 'can't tell'. ${ }^{45}$ This appraisal will provide a structured and thorough analysis the range of quality of studies of knee OA MoCs that has been published, leading to a better interpretation of the results.

\section{Synthesis and presentation of results}

We will perform a descriptive qualitative content analysis that will be presented in a tabular form, aligned with the objectives and research questions of this scoping review. This will include the identification of the MoC, the core intervention components, professionals involved and inherent training, services involved, organisation components, context-related specificities, outcome measures used and outcomes at organisational and patient level and evaluation of implementation success. We will report also as quantitative data, using a descriptive numerical summary, the overall number of studies, study designs, years of publication, types of intervention, characteristics of the study population and geographical distribution of studies. Meta-analysis is not planned.

Additionally, a narrative summary will accompany the tabulated results and will describe how the results relate to the research questions and objectives. As stated before, the data extraction may be updated during the data extraction process.

\section{Consultation with relevant stakeholders}

Two members of the research team (EBC and AMR) have developed and implemented MoCs for musculoskeletal diseases and will be involved throughout the analysis process. This involvement will provide additional sources of information, perspectives, high level of meaning content, expertise and it will enhance the applicability of this scoping study.

\section{Author affiliations}

${ }^{1}$ NOVA National School of Public Health, Public Health Research Centre, Universidade NOVA de Lisboa, Lisbon, Portugal

${ }^{2}$ Comprehensive Health Research Center (CHRC), Universidade Nova de Lisboa, Lisboa, Portugal

${ }^{3}$ EpiDoC Unit - Epidemiology of Chronic Diseases Unit, CEDOC, NOVA Medical School, Universidade Nova de Lisboa, Lisboa, Portugal

${ }^{4}$ Physical Therapy Department, School of Health, Polytechnic Institute of Setúbal, Setúbal, Portugal

${ }^{5}$ Nuffield Department of Population Health, University of Oxford, Oxford, UK

${ }^{6}$ Documentation and Scientific Information Service, Centro Hospitalar e Universitário de Coimbra, Coimbra, Portugal

\section{Twitter Eduardo B Cruz @EBCRUZ64}

Acknowledgements We would like to express our gratitude to Deborah Nossiter for her assistance in the writing process of this article.

Contributors DC, AMR, CN and EBC conceived the study, DC outlined the protocol and drafted this manuscript, DG-B supported and guided the methodology and concepts formulation of the design, DC, LAG, AMR, EBC and CN jointly developed the research questions and inclusion criteria, $\mathrm{HD}$ and DG-B supported the formulation of search strategy. All the authors revised and accepted the final version of the manuscript.

Funding This project is funded by national funds through FCT-Fundação para a Ciência e Tecnologia, I. P. under the PhD grant SFRH/BD/148420/2019 awarded to the first author. This protocol was included in the PhD previously approved project.

Competing interests None declared.
Patient and public involvement statement Patients and/or the public were not involved in the design, or conduct, or reporting, or dissemination plans of this research.

Patient consent for publication Not required.

Provenance and peer review Not commissioned; externally peer reviewed.

Supplemental material This content has been supplied by the author(s). It has not been vetted by BMJ Publishing Group Limited (BMJ) and may not have been peer-reviewed. Any opinions or recommendations discussed are solely those of the author(s) and are not endorsed by BMJ. BMJ disclaims all liability and responsibility arising from any reliance placed on the content. Where the content includes any translated material, BMJ does not warrant the accuracy and reliability of the translations (including but not limited to local regulations, clinical guidelines, terminology, drug names and drug dosages), and is not responsible for any error and/or omissions arising from translation and adaptation or otherwise.

Open access This is an open access article distributed in accordance with the Creative Commons Attribution Non Commercial (CC BY-NC 4.0) license, which permits others to distribute, remix, adapt, build upon this work non-commercially, and license their derivative works on different terms, provided the original work is properly cited, appropriate credit is given, any changes made indicated, and the use is non-commercial. See: http://creativecommons.org/licenses/by-nc/4.0/.

\section{ORCID iDs}

Daniela Costa http://orcid.org/0000-0001-8469-6356

Eduardo B Cruz http://orcid.org/0000-0003-1812-1815

Helena Donato http://orcid.org/0000-0002-1905-1268

\section{REFERENCES}

1 Hunter DJ, Bierma-Zeinstra S. Osteoarthritis. Lancet 2019;393:1745-59

2 Safiri S, Kolahi AA, Hoy D, et al. Global, regional and national burden of rheumatoid arthritis 1990-2017: a systematic analysis of the global burden of disease study 2017. Ann Rheum Dis 2019;78:1463-71.

3 Martel-Pelletier J, Maheu E, Pelletier J-P, et al. A new decision tree for diagnosis of osteoarthritis in primary care: international consensus of experts. Aging Clin Exp Res 2019;31:19-30.

4 Parmelee PA, Tighe CA, Dautovich ND. Sleep disturbance in osteoarthritis: linkages with pain, disability, and depressive symptoms. Arthritis Care Res 2015;67:358-65.

5 Puig-Junoy J, Ruiz Zamora A. Socio-economic costs of osteoarthritis: a systematic review of cost-of-illness studies. Semin Arthritis Rheum 2015;44:531-41.

6 Salmon JH, Rat AC, Sellam J, et al. Economic impact of lower-limb osteoarthritis worldwide: a systematic review of cost-of-illness studies. Osteoarthr Cartil 2016;24:1500-8.

7 Turkiewicz A, Petersson IF, Björk J, et al. Current and future impact of osteoarthritis on health care: a population-based study with projections to year 2032. Osteoarthr Cartilage 2014;22:1826-32.

8 Bannuru RR, Osani MC, Vaysbrot EE, et al. OARSI guidelines for the non-surgical management of knee, hip, and polyarticular osteoarthritis. Osteoarthritis Cartilage 2019;27:1578-89.

9 Kolasinski SL, Neogi T, Hochberg MC, et al. 2019 American College of Rheumatology/Arthritis Foundation guideline for the management of osteoarthritis of the hand, hip, and knee. Arthritis Rheumatol 2020;72:220-33.

10 Salmon JH, Rat AC, Sellam J, et al. Economic impact of lower-limb osteoarthritis worldwide: a systematic review of cost-of-illness studies. Osteoarthritis Cartilage 2016;24:1500-8.

11 Belmont PJ, Goodman GP, Waterman BR, et al. Thirty-day postoperative complications and mortality following total knee arthroplasty: incidence and risk factors among a national sample of 15,321 patients. J Bone Joint Surg Am 2014;96:20-6.

12 Skou ST, Roos EM, Laursen MB. A randomized, controlled trial of total knee replacement. N Engl J Med 2016;374:692.

13 Berwick D, Snair M, Nishtar S. Crossing the global health care quality chasm: a key component of universal health coverage. JAMA 2018;320:1317-8.

14 Price AJ, Alvand A, Troelsen A, et al. Knee replacement. Lancet 2018;392:1672-82.

15 Hunt LP, Ben-Shlomo Y, Clark EM, et al. 45-day mortality after 467,779 knee replacements for osteoarthritis from the National joint Registry for England and Wales: an observational study. Lancet 2014;384:1429-36.

16 Basedow M, Esterman A. Assessing appropriateness of osteoarthritis care using quality indicators: a systematic review. J Eval Clin Pract 2015;21:782-9. 
17 Edwards JJ, Khanna M, Jordan KP, et al. Quality indicators for the primary care of osteoarthritis: a systematic review. Ann Rheum Dis 2015;74:490-8.

18 Pabinger $\mathrm{C}$, Lothaller $\mathrm{H}$, Geissler A. Utilization rates of kneearthroplasty in OECD countries. Osteoarthritis Cartilage 2015;23:1664-73.

19 Egerton T, Diamond LE, Buchbinder R, et al. A systematic review and evidence synthesis of qualitative studies to identify primary care clinicians' barriers and enablers to the management of osteoarthritis. Osteoarthritis Cartilage 2017;25:625-38.

20 Hunter DJ, Ther J. Osteoarthritis management: time to change the Deck. J Orthop Sports Phys Ther 2017;47:370-2.

21 Bruyère $\mathrm{O}$, Honvo $\mathrm{G}$, Veronese $\mathrm{N}$, et al. An updated algorithm recommendation for the management of knee osteoarthritis from the European Society for clinical and economic aspects of osteoporosis, osteoarthritis and musculoskeletal diseases (ESCEO). Semin Arthritis Rheum 2019;49:337-50.

22 Briggs AM, Towler SCB, Speerin R, et al. Models of care for musculoskeletal health in Australia: now more than ever to drive evidence into health policy and practice. Aust Health Rev 2014;38:401-5

23 Briggs AM, Chan M, Slater $\mathrm{H}$. Models of care for musculoskeletal health: moving towards meaningful implementation and evaluation across conditions and care settings. Best Pract Res Clin Rheumatol 2016;30:359-74.

24 Thorstensson CA, Garellick G, Rystedt H, et al. Better management of patients with osteoarthritis: development and nationwide implementation of an evidence-based supported osteoarthritis selfmanagement programme. Musculoskeletal Care 2015;13:67-75.

25 Jordan KP, Edwards JJ, Porcheret M, et al. Effect of a model consultation informed by guidelines on recorded quality of care of osteoarthritis (MOSAICS): a cluster randomised controlled trial in primary care. Osteoarthritis Cartilage 2017;25:1588-97.

26 Skou ST, Roos EM. Good Life with osteoArthritis in Denmark (GLA:D ${ }^{\mathrm{TM}}$ ): evidence-based education and supervised neuromuscular exercise delivered by certified physiotherapists nationwide. BMC Musculoskelet Disord 2017;18:72

27 Smink AJ, van den Ende CHM, Vliet Vlieland TPM, et al. "Beating osteoARThritis": development of a stepped care strategy to optimize utilization and timing of non-surgical treatment modalities for patients with hip or knee osteoarthritis. Clin Rheumatol 2011;30:1623-9.

28 Eyles JP, Hunter DJ, Bennell KL, et al. Priorities for the effective implementation of osteoarthritis management programs: an OARSI international consensus exercise. Osteoarthritis Cartilage 2019;27:1270-9.

29 Allen KD, Choong PF, Davis AM, et al. Osteoarthritis: models for appropriate care across the disease continuum. Best Pract Res Clin Rheumatol 2016;30:503-35.
30 Coles E, Anderson J, Maxwell M, et al. The influence of contextual factors on healthcare quality improvement initiatives: a realist review. Syst Rev 2020;9:94.

31 Levac D, Colquhoun H, O'Brien KK. Scoping studies: advancing the methodology. Implement Sci 2010;5:69.

32 Colquhoun HL, Levac D, O'Brien KK, et al. Scoping reviews: time for clarity in definition, methods, and reporting. J Clin Epidemiol 2014;67:1291-4.

33 Arksey H, O'Malley L. Scoping studies: towards a methodological framework. Int J Soc Res Methodol 2005;8:19-32.

34 Munn Z, Peters MDJ, Stern C, et al. Systematic review or scoping review? Guidance for authors when choosing between a systematic or scoping review approach. BMC Med Res Methodol 2018;18:1-7.

35 The Joanna Briggs Institute. Joanna Briggs Institute Reviewers' Manual: 2015 edition / Supplement;2015.

36 Tricco AC, Lillie E, Zarin W, et al. PRISMA extension for scoping reviews (PRISMA-ScR): checklist and explanation. Ann Intern Med 2018;169:467-73.

37 Briggs AM, Jordan JE, Jennings M. A framework to evaluate musculoskeletal models of care. Cornwall, UK, 2016.

38 World Health Organization. Primary health care: closing the gap between public health and primary care through integration. Astana, 2018.

39 Eldh AC, Almost J, DeCorby-Watson K, et al. Clinical interventions, implementation interventions, and the potential greyness in between -A discussion paper. BMC Health Serv Res 2017;17:1-10.

40 Palmer K, Marengoni A, Forjaz MJ, et al. Multimorbidity care model: recommendations from the consensus meeting of the joint action on chronic diseases and promoting healthy ageing across the life cycle (JA-CHRODIS). Health Policy 2018;122:4-11.

41 Lefebvre C, Glanville J, Briscoe S. Chapter 4: Searching for and selecting studies. In: Cochrane Handbook for systematic reviews of interventions version 6.0. Cochrane, ed, 2019.

42 Pham MT, Rajić A, Greig JD, et al. A scoping review of scoping reviews: advancing the approach and enhancing the consistency. Res Synth Methods 2014;5:371-85.

43 Daudt HML, van Mossel C, Scott SJ. Enhancing the scoping study methodology: a large, inter-professional team's experience with Arksey and O'Malley's framework. BMC Med Res Methodol 2013;13:1-9.

44 Grant MJ, Booth A. A typology of reviews: an analysis of 14 review types and associated methodologies. Health Info Libr J 2009;26:91-108.

45 Hong QN, Pluye P, Fàbregues S, et al. Improving the content validity of the mixed methods appraisal tool: a modified e-Delphi study. $J$ Clin Epidemiol 2019;111:49-59. 